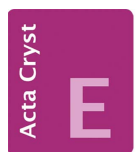

CRYSTALLOGRAPHIC COMMUNICATIONS

ISSN 2056-9890

Received 18 November 2016

Accepted 28 November 2016

Edited by M. Weil, Vienna University of Technology, Austria

Keywords: crystal structure; silver; argentophilic interactions; fluorides; ammine ligand.

CCDC reference: 1519625

Supporting information: this article has supporting information at journals.iucr.org/e

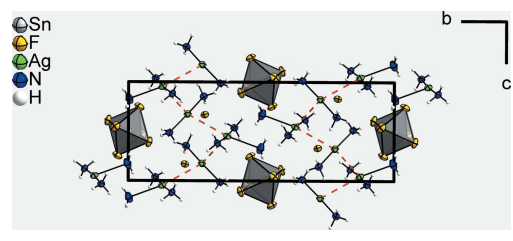

OPEN $\odot$ ACCESS

\section{Crystal structure of $\left[\mathrm{Ag}\left(\mathrm{NH}_{3}\right)_{3}\right]_{2}\left[\mathrm{Ag}\left(\mathrm{NH}_{3}\right)_{2}\right]_{2}\left[\mathrm{SnF}_{6}\right] \mathrm{F}_{2}$, a compound showing argentophilic interactions}

\author{
Florian Kraus, ${ }^{*}$ Matthias Fichtl and Sebastian Baer
}

Anorganische Chemie, Fluorchemie, Fachbereich Chemie, Philipps-Universität Marburg, Hans-Meerwein-Strasse 4, 35032 Marburg, Germany. *Correspondence e-mail: florian.kraus@chemie.uni-marburg.de

Bis[triamminesilver(I)] bis[diamminesilver(I)] hexafluoridostannate(IV) difluoride, $\left[\mathrm{Ag}\left(\mathrm{NH}_{3}\right)_{3}\right]_{2}\left[\mathrm{Ag}\left(\mathrm{NH}_{3}\right)_{2}\right]_{2}\left[\mathrm{SnF}_{6}\right] \mathrm{F}_{2}$, was obtained in the form of colourless crystals from the reaction of $\mathrm{CsAgSnF}_{7}$ in anhydrous ammonia. Two different ammine complexes of silver(I) are present in the structure, i.e. a linear diammine and a T-shaped triammine complex. The ammine silver(I) complexes show Ag...Ag distances in the range of argentophilic interactions. In the crystal, several $\mathrm{N}-\mathrm{H} \cdots \mathrm{F}$ hydrogen bonds are present between the complex cations and the $\mathrm{SbF}_{6}^{-}$and $\mathrm{F}^{-}$anions, leading to the formation of a three-dimensional network.

\section{Chemical context}

Metallophilicity, especially argento- and aurophilicity, is a theoretically and experimentally well-established concept, see, for example, the seminal works of Jansen (Jansen, 1987), Schmidbaur and co-workers (Scherbaum et al., 1988; Schmidbaur, 1995; Schmidbaur \& Schier, 2012, 2015) or Pyykkö and co-workers (Pyykkö \& Zhao, 1991; Pyykkö, 1997,2004; Pyykkö et al., 1997; Pyykkö \& Mendizabal, 1997). We reacted a silver(II) compound, $\mathrm{CsAgSnF}_{7}$, with anhydrous

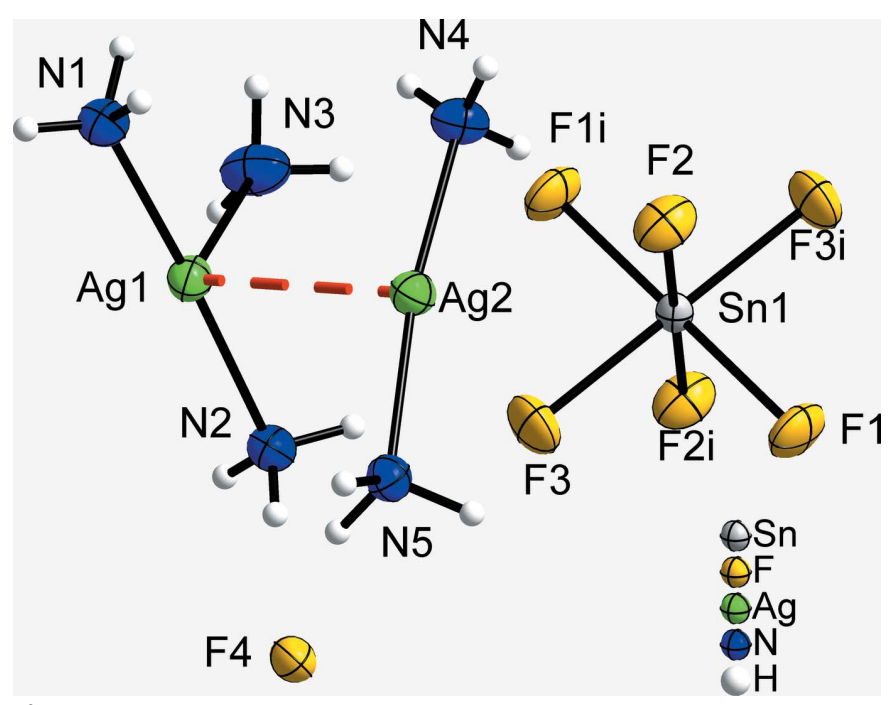

Figure 1

The principal building units in the crystal structure of the title compound, showing the $\mathrm{F}^{-}$anion, the $\left[\mathrm{SnF}_{6}\right]^{2-}$ anion, as well as the argentophilic interaction (in red) between the $\left[\mathrm{Ag}\left(\mathrm{NH}_{3}\right)_{2}\right]^{+}$and $\left[\mathrm{Ag}\left(\mathrm{NH}_{3}\right)_{3}\right]^{+}$cations. Displacement ellipsoids are drawn at the $70 \%$ probability level and $\mathrm{H}$ atoms are shown with an arbitrary radius. [Symmetry code: (i) $-x+1,-y$, $-z+1$.] 


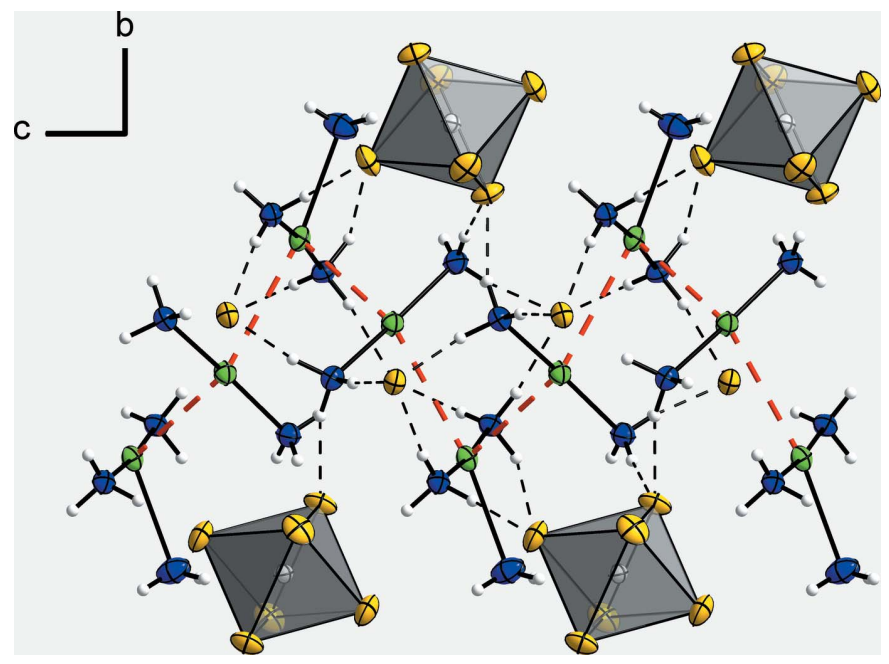

Figure 2

A section of the crystal structure in a view along [100], showing a corrugated strand of complex cations running along [001]. The argentophilic interactions are drawn as red dashed bonds between the $\mathrm{Ag}^{\mathrm{I}}$ atoms and $\mathrm{N}-\mathrm{H} \cdots \mathrm{F}$ hydrogen bonds are shown as dashed lines. $\left[\mathrm{SnF}_{6}\right]^{2-}$ anions are shown as polyhedra to highlight their positions relative to the the kinks of the strand. Displacement ellipsoids are as in Fig. 1.

liquid ammonia and observed the reduction of $\mathrm{Ag}^{\mathrm{II}}$. The preparation conditions and crystal structure of the thus obtained $\mathrm{Ag}^{\mathrm{I}}$ title compound, $\left[\mathrm{Ag}\left(\mathrm{NH}_{3}\right)_{3}\right]_{2}\left[\mathrm{Ag}\left(\mathrm{NH}_{3}\right)_{2}\right]_{2}-$ $\left[\mathrm{SnF}_{6}\right] \mathrm{F}_{2}$, is reported here. The short $\mathrm{Ag} \cdots \mathrm{Ag}$ distances between the complex cations are in the range of argentophilic interactions.

\section{Structural commentary}

$\left[\mathrm{Ag}\left(\mathrm{NH}_{3}\right)_{3}\right]_{2}\left[\mathrm{Ag}\left(\mathrm{NH}_{3}\right)_{2}\right]_{2}\left[\mathrm{SnF}_{6}\right] \mathrm{F}_{2}$ crystallizes in space group type $P 2_{1} / c$. The Sn atom occupies Wyckoff position $2 d$ (site

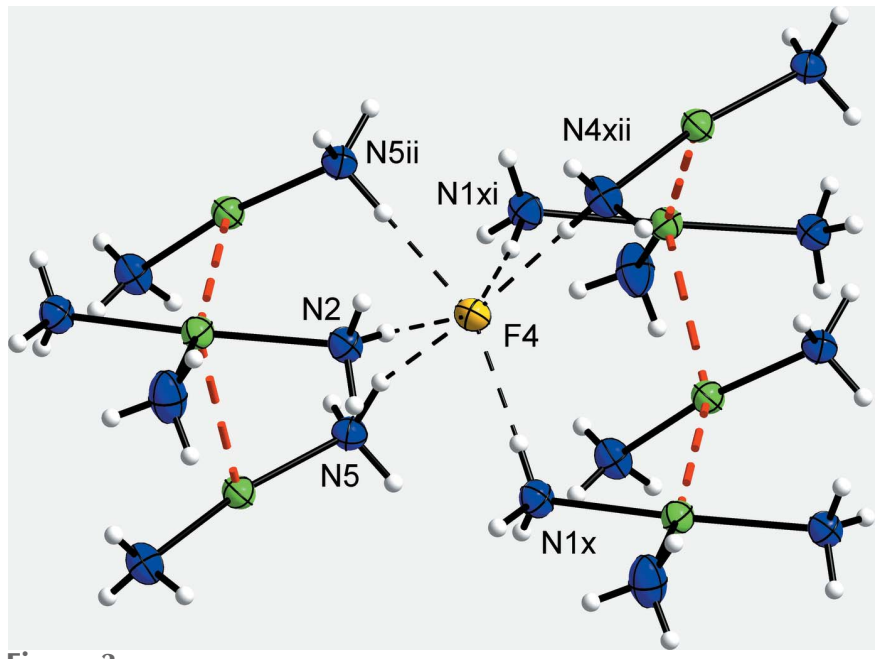

Figure 3

A section of the crystal structure of the title compound, showing the $\mathrm{N}-$ $\mathrm{H}$...F hydrogen bonds (dashed lines) around the free fluoride anion and the bridging of the corrugated $\mathrm{Ag} \cdots \mathrm{Ag}$ strands (red dashed lines). Displacement ellipsoids are as in Fig. 1. [Symmetry codes: (ii) $x,-y+\frac{1}{2}$, $z+\frac{1}{2} ;(x) 1+x, y, z ;(x i) 1+x, \frac{1}{2}-y, \frac{1}{2}+z ;($ xii) $1+x, y, 1+z$.]

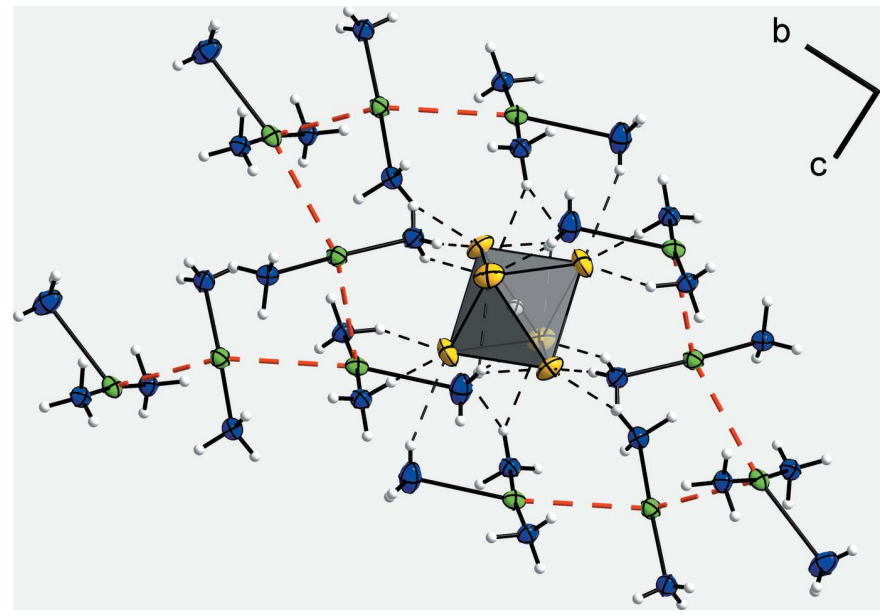

Figure 4

A section of the crystal structure, showing the hydrogen bonding towards the $\left[\mathrm{SnF}_{6}\right]^{2-}$ anion, which is shown as a polyhedron. $\mathrm{Ag}^{\mathrm{I}}$ atoms are interconnected by $\mathrm{Ag} \cdots \mathrm{Ag}$ interactions (red dashed lines) to show the formation of strands. Displacement ellipsoids are as in Fig. 1.

symmetry $\overline{1})$, all other atoms reside on general positions $4 e$. The structure comprises of $\left[\mathrm{Ag}\left(\mathrm{NH}_{3}\right)_{3}\right]^{+}$and $\left[\mathrm{Ag}\left(\mathrm{NH}_{3}\right)_{2}\right]^{+}$ complex cations as well as $\mathrm{F}^{-}$and $\left[\mathrm{SnF}_{6}\right]^{2-}$ anions (Fig. 1). The diamminesilver(I) cation $(\mathrm{Ag} 2)$ is almost linear with an $\mathrm{N}-$ $\mathrm{Ag}-\mathrm{N}$ angle of $170.93(7)^{\circ}$ and $\mathrm{Ag}-\mathrm{N}$ distances of 2.1160 (16) and 2.1183 (16) $\AA$. The deviation from linearity is likely to arise from the surrounding, i.e. $\mathrm{N}-\mathrm{H} \cdots \mathrm{F}$ hydrogen bonding to adjacent $\left[\mathrm{SnF}_{6}\right]^{2-}$ and $\mathrm{F}^{-}$anions. This $\left[\mathrm{Ag}\left(\mathrm{NH}_{3}\right)_{2}\right]^{+}$ cation shows a short $\mathrm{Ag} \cdots \mathrm{Ag}$ distance of 3.0611 (2) $\AA$ to a neighboring $\left[\mathrm{Ag}\left(\mathrm{NH}_{3}\right)_{3}\right]^{+}$cation and another slightly longer $\mathrm{Ag} \cdots \mathrm{Ag}$ distance of $3.3282(2) \AA$ to a second $\left[\mathrm{Ag}\left(\mathrm{NH}_{3}\right)_{3}\right]^{+}$ cation (symmetry code $x,-y+\frac{1}{2}, z+\frac{1}{2}$ ). The triammine silver(I) cation $(\mathrm{Ag} 1)$ is $\mathrm{T}$-shaped and can be viewed as a linear diammine silver(I) cation to which another ammine ligands is bound at a longer distance. The short $\mathrm{Ag}-\mathrm{N}$ distances are 2.1434 (16) and 2.1662 (16) $\AA$, and the remote ammine ligand is bound at a distance of 2.5870 (19) $\AA$. The $\mathrm{N}-\mathrm{Ag}-\mathrm{N}$ angle between the shortly bonded ligands is $173.74(7)^{\circ}$. The deviation of $\mathrm{N}-\mathrm{Ag}-\mathrm{N}$ angles including the remote ammine ligand from $90^{\circ}\left[85.44(6)\right.$ and $\left.110.82(6)^{\circ}\right]$ are probably due to hydrogen bonding of the ammine ligands to $\mathrm{F}$ atoms of the anions.

\section{Supramolecular features}

As a result of the short $\mathrm{Ag} 1 \cdots \mathrm{Ag} 2$ contacts, corrugated strands of alternating $\left[\mathrm{Ag}\left(\mathrm{NH}_{3}\right)_{3}\right]^{+}$and $\left[\mathrm{Ag}\left(\mathrm{NH}_{3}\right)_{2}\right]^{+}$cations occur where the $\left[\mathrm{Ag}\left(\mathrm{NH}_{3}\right)_{3}\right]^{+}$cations form the kinks which are connected by the $\left[\mathrm{Ag}\left(\mathrm{NH}_{3}\right)_{2}\right]^{+}$cations. The strands run parallel to the $c$ axis (Fig. 2). Similar metallophilic interactions have been observed in the ammine copper(I) fluoride $\left\{\left[\mathrm{Cu}\left(\mathrm{NH}_{3}\right)_{3}\right]_{2}\left[\mathrm{Cu}_{2}\left(\mathrm{NH}_{3}\right)_{4}\right]\right\} \mathrm{F}_{4} \cdot 4 \mathrm{NH}_{3}$ (Woidy et al., 2015). However, the cuprophilic interactions are only observed between the diammine copper(I) cations forming linear strands whereas the triammine copper(I) cations do not show such interactions. 
Table 1

Hydrogen-bond geometry $\left(\AA,^{\circ}\right)$.

\begin{tabular}{lllll}
\hline$D-\mathrm{H} \cdots A$ & $D-\mathrm{H}$ & $\mathrm{H} \cdots A$ & $D \cdots A$ & $D-\mathrm{H} \cdots A$ \\
\hline $\mathrm{N} 1-\mathrm{H} 1 A \cdots \mathrm{F} 4^{\mathrm{i}}$ & $0.85(3)$ & $2.03(3)$ & $2.884(2)$ & $178(2)$ \\
$\mathrm{N} 1-\mathrm{H} 1 B \cdots \mathrm{F} 4^{\mathrm{ii}}$ & $0.89(3)$ & $1.96(3)$ & $2.844(2)$ & $171(3)$ \\
$\mathrm{N} 1-\mathrm{H} 1 C \cdots \mathrm{F} 3^{\mathrm{i}}$ & $0.98(4)$ & $2.14(4)$ & $3.057(2)$ & $156(3)$ \\
$\mathrm{N} 2-\mathrm{H} 2 A \cdots \mathrm{F} 1^{\mathrm{iii}}$ & $0.91(3)$ & $2.43(3)$ & $3.227(2)$ & $146(2)$ \\
$\mathrm{N} 2-\mathrm{H} 2 A \cdots \mathrm{F} 3^{\text {iv }}$ & $0.91(3)$ & $2.56(3)$ & $3.354(2)$ & $145.4(19)$ \\
$\mathrm{N} 2-\mathrm{H} 2 B \cdots \mathrm{F} 3$ & $0.94(3)$ & $2.04(3)$ & $2.961(2)$ & $167(3)$ \\
$\mathrm{N} 2-\mathrm{H} 2 C \cdots \mathrm{F} 4$ & $0.83(3)$ & $2.02(3)$ & $2.849(2)$ & $172(3)$ \\
$\mathrm{N} 3-\mathrm{H} 3 A \cdots \mathrm{F} 1^{\mathrm{i}}$ & $0.88(3)$ & $2.57(3)$ & $3.274(2)$ & $138(3)$ \\
$\mathrm{N} 3-\mathrm{H} 3 A \cdots \mathrm{F} 2^{\mathrm{v}}$ & $0.88(3)$ & $2.42(3)$ & $3.223(2)$ & $153(3)$ \\
$\mathrm{N} 3-\mathrm{H} 3 B \cdots \mathrm{F} 3^{\text {iv }}$ & $0.79(3)$ & $2.61(3)$ & $3.345(3)$ & $157(3)$ \\
$\mathrm{N} 3-\mathrm{H} 3 C \cdots \mathrm{F} 1^{\mathrm{vi}}$ & $0.91(3)$ & $2.39(3)$ & $3.279(3)$ & $167(3)$ \\
$\mathrm{N} 4-\mathrm{H} 4 A \cdots \mathrm{F} 2$ & $0.90(3)$ & $2.04(3)$ & $2.930(2)$ & $170(2)$ \\
$\mathrm{N} 4-\mathrm{H} 4 B \cdots \mathrm{F} 4^{\text {vii }}$ & $0.84(3)$ & $1.97(3)$ & $2.7955(19)$ & $171(3)$ \\
$\mathrm{N} 4-\mathrm{H} 4 C \cdots \mathrm{F} 1^{\mathrm{i}}$ & $0.79(3)$ & $2.33(3)$ & $3.045(2)$ & $151(3)$ \\
$\mathrm{N} 5-\mathrm{H} 5 A \cdots \mathrm{F} 4$ & $0.96(3)$ & $1.90(3)$ & $2.8305(19)$ & $160(3)$ \\
$\mathrm{N} 5-\mathrm{H} 5 B \cdots \mathrm{F} 4^{\text {viii }}$ & $0.95(3)$ & $1.93(3)$ & $2.882(2)$ & $173(2)$ \\
$\mathrm{N} 5-\mathrm{H} 5 C \cdots \mathrm{F} 2^{\text {ix }}$ & $0.93(3)$ & $2.09(3)$ & $2.999(2)$ & $163(3)$ \\
\hline
\end{tabular}

Symmetry codes: (i) $x-1, y, z$; (ii) $x-1,-y+\frac{1}{2}, z-\frac{1}{2}$; (iii) $x, y, z+1$; (iv) $-x+1,-y,-z+2$; (v) $-x,-y,-z+1$; (vi) $-x+1,-y,-z+1$; (vii) $x-1, y, z-1$; (viii) $x,-y+\frac{1}{2}, z-\frac{1}{2}$; (ix) $x,-y+\frac{1}{2}, z+\frac{1}{2}$.

In the title structure, the fluoride anions reside above and below the cation strands and connect neighbouring strands via $\mathrm{N}-\mathrm{H} \cdots \mathrm{F}$ hydrogen bonds, whereas the $\left[\mathrm{SnF}_{6}\right]^{2-}$ anions lie on the sides of the strands, also connecting neighbouring ones. The free fluoride ion (F4) is an acceptor of six hydrogen bonds (Fig. 3). Its coordination environment resembles an octahedron with one longer edge. It interconnects the $\mathrm{Ag} \cdots \mathrm{Ag}$ strands along the $a$-axis. The $\left[\mathrm{SnF}_{6}\right]^{2-}$ anion interconnects four of the Ag...Ag strands (Fig. 4). Four of the six F atoms bonded to the $\mathrm{Sn}$ atom are acceptors of four hydrogen bonds (two regular, two bifurcated), the other two $\mathrm{F}$ atoms are acceptors of three hydrogen bonds. The diammine silver(I) cations only form regular hydrogen bonds, whereas the
Table 2

Experimental details.

\begin{tabular}{|c|c|}
\hline \multicolumn{2}{|l|}{ Crystal data } \\
\hline Chemical formula & {$\left[\mathrm{Ag}\left(\mathrm{NH}_{3}\right)_{3}\right]_{2}\left[\mathrm{Ag}\left(\mathrm{NH}_{3}\right)_{2}\right]_{2}\left[\mathrm{SnF}_{6}\right] \mathrm{F}_{2}$} \\
\hline$M_{\mathrm{r}}$ & 872.51 \\
\hline Crystal system, space group & Monoclinic, $P 2_{1} / c$ \\
\hline Temperature (K) & 123 \\
\hline$a, b, c(\AA)$ & $7.3274(2), 19.4495$ (4), 7.8579 (3) \\
\hline$\beta\left(^{\circ}\right)$ & $113.205(4)$ \\
\hline$V\left(\AA^{3}\right)$ & $1029.27(6)$ \\
\hline$Z$ & 2 \\
\hline Radiation type & Mo $K \alpha$ \\
\hline$\mu\left(\mathrm{mm}^{-1}\right)$ & 5.01 \\
\hline Crystal size $(\mathrm{mm})$ & $0.20 \times 0.05 \times 0.05$ \\
\hline
\end{tabular}

Data collection

Diffractometer

Absorption correction

$T_{\min }, T_{\max }$

No. of measured, independent and observed $[I>2 \sigma(I)]$ reflections

$R_{\text {int }}$

$(\sin \theta / \lambda)_{\max }\left(\AA^{-1}\right)$
Oxford-Diffraction Xcalibur3
Multi-scan (CrysAlis RED; Oxford Diffraction, 2008)
$0.602,1.000$
$30418,5731,4330$
0.030
0.889
$0.020,0.046,0.99$
5731
167
All $\mathrm{H}$-atom parameters refined
$1.04,-0.93$
Refinement
$R\left[F^{2}>2 \sigma\left(F^{2}\right)\right], w R\left(F^{2}\right), S$
No. of reflections
No. of parameters
$\mathrm{H}$-atom treatment
$\Delta \rho_{\max }, \Delta \rho_{\min }\left(\mathrm{e} \AA^{-3}\right)$

Computer programs: CrysAlis CCD and CrysAlis RED (Oxford Diffraction, 2008), SHELXS97 (Sheldrick, 2008), SHELXL2016 (Sheldrick, 2015), SHELXLE (Hübschle et al., 2011), DIAMOND (Brandenburg, 2012) and publCIF (Westrip, 2010).

triammine silver(I) cations form regular as well as bifurcated hydrogen bonds. The bifurcated hydrogen bonds bridge four edges of each $\left[\mathrm{SnF}_{6}\right]^{2-}$ octahedron. Overall, a rather complex three-dimensional hydrogen-bonded network results (Fig. 5). Numerical details of the hydrogen-bonding interactions are summarized in Table 1.

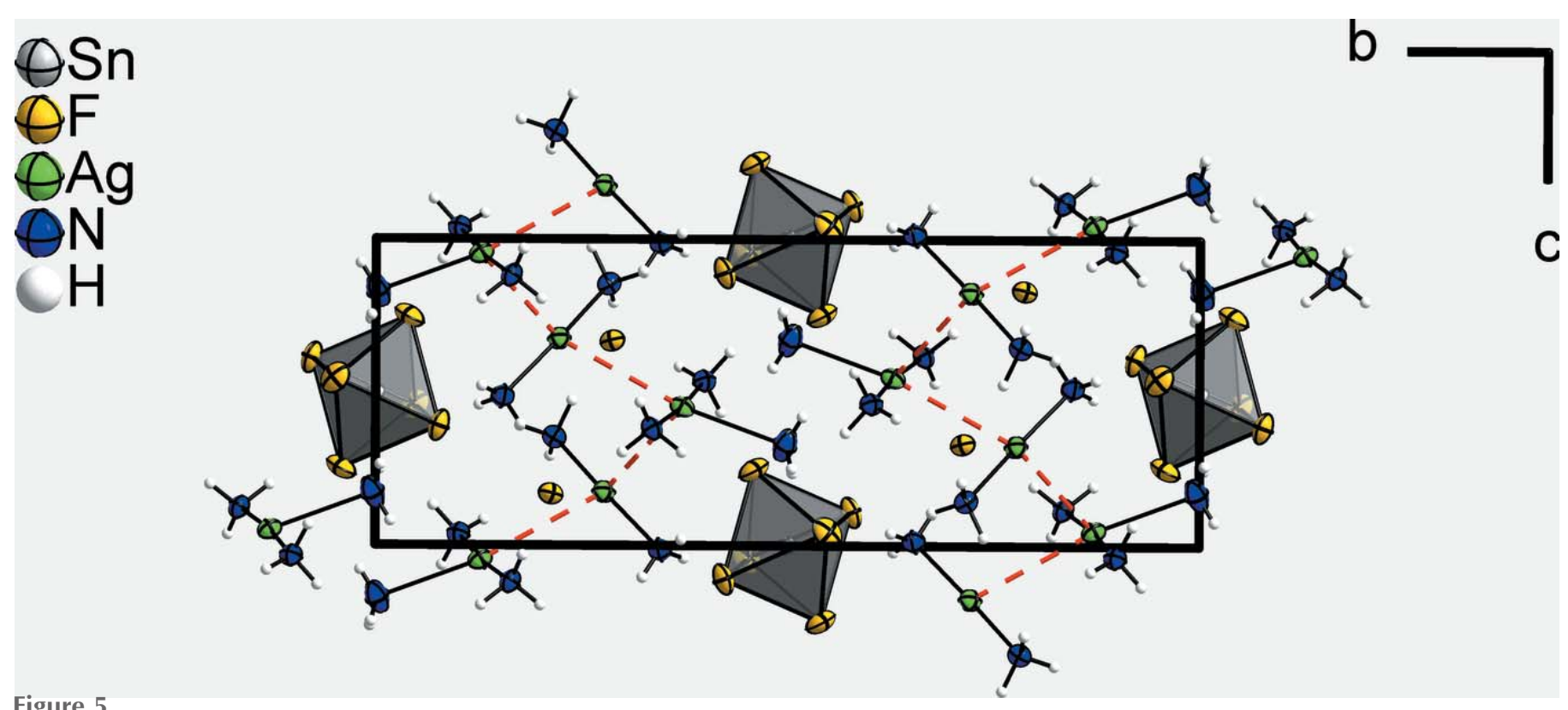

The crystal structure of the title compound. $\mathrm{Ag}^{\mathrm{I}}$ atoms are interconnected by argentophilic interactions (red dashed lines) to show the formation of strands and $\left[\mathrm{SnF}_{6}\right]^{2-}$ anions are shown as polyhedra. Displacement ellipsoids are as in Fig. 1. 


\section{Synthesis and crystallization}

$870 \mathrm{mg}$ of $\mathrm{CsAgSnF}_{7}$ were reacted with approximately $10 \mathrm{ml}$ of anhydrous liquid ammonia at $195 \mathrm{~K}$. Upon contact, the greenish colour of the educt vanished and a white powder was observed. This indicates that $\mathrm{Ag}^{\mathrm{II}}$ was reduced to $\mathrm{Ag}^{\mathrm{I}}$ and ammonia was oxidized to $\mathrm{N}_{2}$. From this white powder, colorless crystals grew within three months of storage at $233 \mathrm{~K}$ of which a suitable one was selected for the diffraction experiment. The role of the Cs atoms remains unclear.

\section{Refinement}

Crystal data, data collection and structure refinement details are summarized in Table 2. Hydrogen atoms were localized from difference Fourier syntheses and were refined freely.

\section{Acknowledgements}

FK thanks the DFG for a Heisenberg Professorship and Dr Matthias Conrad for helpful discussions.

\section{References}

Brandenburg, K. (2012). DIAMOND. Crystal Impact GbR, Bonn, Germany.

Hübschle, C. B., Sheldrick, G. M. \& Dittrich, B. (2011). J. Appl. Cryst. 44, 1281-1284.

Jansen, M. (1987). Angew. Chem. Int. Ed. Engl. 26, 1098-1110.

Oxford Diffraction (2008). CrysAlis CCD and CrysAlis RED. Oxford Diffraction Ltd, Abingdon, Oxfordshire, England.

Pyykkö, P. (1997). Chem. Rev. 97, 597-636.

Pyykkö, P. (2004). Angew. Chem. Int. Ed. 43, 4412-4456.

Pyykkö, P. \& Mendizabal, F. (1997). Chem. Eur. J. 3, 1458-1465.

Pyykkö, P., Runeberg, N. \& Mendizabal, F. (1997). Chem. Eur. J. 3, 1451-1457.

Pyykkö, P. \& Zhao, Y. (1991). Angew. Chem. Int. Ed. Engl. 30, 604605.

Scherbaum, F., Grohmann, A., Huber, B., Krüger, C. \& Schmidbaur, H. (1988). Angew. Chem. Int. Ed. Engl. 27, 1544-1546.

Schmidbaur, H. (1995). Chem. Soc. Rev. 24, 391-400.

Schmidbaur, H. \& Schier, A. (2012). Chem. Soc. Rev. 41, 370-412.

Schmidbaur, H. \& Schier, A. (2015). Angew. Chem. Int. Ed. 54, 746784.

Sheldrick, G. M. (2008). Acta Cryst. A64, 112-122.

Sheldrick, G. M. (2015). Acta Cryst. C71, 3-8.

Westrip, S. P. (2010). J. Appl. Cryst. 43, 920-925.

Woidy, P., Karttunen, A. J., Widenmeyer, M., Niewa, R. \& Kraus, F. (2015). Chem. Eur. J. 21, 3290-3303. 


\section{supporting information}

Acta Cryst. (2016). E72, 1860-1863 [https://doi.org/10.1107/S2056989016019010]

\section{Crystal structure of $\left[\mathrm{Ag}\left(\mathrm{NH}_{3}\right)_{3}\right]_{2}\left[\mathrm{Ag}\left(\mathrm{NH}_{3}\right)_{2}\right]_{2}\left[\mathrm{SnF}_{6}\right] \mathrm{F}_{2}$, a compound showing argentophilic interactions}

\section{Florian Kraus, Matthias Fichtl and Sebastian Baer}

\section{Computing details}

Data collection: CrysAlis CCD (Oxford Diffraction, 2008); cell refinement: CrysAlis RED (Oxford Diffraction, 2008); data reduction: CrysAlis RED (Oxford Diffraction, 2008); program(s) used to solve structure: SHELXS97 (Sheldrick, 2008); program(s) used to refine structure: SHELXL2016 (Sheldrick, 2015) and SHELXLE (Hübschle et al., 2011); molecular graphics: DIAMOND (Brandenburg, 2012); software used to prepare material for publication: publCIF (Westrip, 2010).

Bis[triamminesilver(I)] bis[diamminesilver(I)] hexafluoridostannate(IV) difluoride

Crystal data

$\left[\mathrm{Ag}\left(\mathrm{NH}_{3}\right)_{3}\right]_{2}\left[\mathrm{Ag}\left(\mathrm{NH}_{3}\right)_{2}\right]_{2}\left[\mathrm{SnF}_{6}\right] \mathrm{F}_{2}$

$M_{r}=872.51$

Monoclinic, $P 2_{1} / c$

$a=7.3274(2) \AA$

$b=19.4495(4) \AA$

$c=7.8579(3) \AA$

$\beta=113.205(4)^{\circ}$

$V=1029.27(6) \AA^{3}$

$Z=2$

Data collection

Oxford-Diffraction Xcalibur3 diffractometer

Radiation source: Enhance (Mo) X-ray Source

Graphite monochromator

Detector resolution: 16.0238 pixels $\mathrm{mm}^{-1}$

phi- and $\omega$-rotation scans

Absorption correction: multi-scan

(CrysAlis RED; Oxford Diffraction, 2008)

$T_{\min }=0.602, T_{\max }=1.000$

\section{Refinement}

Refinement on $F^{2}$

Least-squares matrix: full

$R\left[F^{2}>2 \sigma\left(F^{2}\right)\right]=0.020$

$w R\left(F^{2}\right)=0.046$

$S=0.99$

5731 reflections

167 parameters

0 restraints
$F(000)=820$

$D_{\mathrm{x}}=2.815 \mathrm{Mg} \mathrm{m}^{-3}$

Mo $K \alpha$ radiation, $\lambda=0.71073 \AA$

Cell parameters from 16653 reflections

$\theta=2.8-39.1^{\circ}$

$\mu=5.01 \mathrm{~mm}^{-1}$

$T=123 \mathrm{~K}$

Block, colorless

$0.20 \times 0.05 \times 0.05 \mathrm{~mm}$

30418 measured reflections

5731 independent reflections

4330 reflections with $I>2 \sigma(I)$

$R_{\text {int }}=0.030$

$\theta_{\text {max }}=39.2^{\circ}, \theta_{\min }=3.0^{\circ}$

$h=-12 \rightarrow 13$

$k=-34 \rightarrow 30$

$l=-13 \rightarrow 13$

Primary atom site location: structure-invariant direct methods

Secondary atom site location: difference Fourier map

Hydrogen site location: difference Fourier map

All $\mathrm{H}$-atom parameters refined

$w=1 /\left[\sigma^{2}\left(F_{0}^{2}\right)+(0.0215 P)^{2}\right]$

where $P=\left(F_{\mathrm{o}}^{2}+2 F_{\mathrm{c}}^{2}\right) / 3$ 
$(\Delta / \sigma)_{\max }=0.002$

$\Delta \rho_{\max }=1.04 \mathrm{e} \AA^{-3}$

$\Delta \rho_{\min }=-0.93$ e $\AA^{-3}$
Extinction correction: SHELXL2016

(Sheldrick, 2015),

$\mathrm{Fc}^{*}=\mathrm{kFc}\left[1+0.001 \mathrm{xFc}^{2} \lambda^{3} / \sin (2 \theta)\right]^{-1 / 4}$

Extinction coefficient: 0.00277 (14)

\section{Special details}

Geometry. All esds (except the esd in the dihedral angle between two 1.s. planes) are estimated using the full covariance matrix. The cell esds are taken into account individually in the estimation of esds in distances, angles and torsion angles; correlations between esds in cell parameters are only used when they are defined by crystal symmetry. An approximate (isotropic) treatment of cell esds is used for estimating esds involving l.s. planes.

Fractional atomic coordinates and isotropic or equivalent isotropic displacement parameters $\left(\AA^{2}\right)$

\begin{tabular}{lllll}
\hline & $x$ & $y$ & $z$ & $U_{\text {iso }} / U_{\text {eq }}$ \\
\hline SN1 & 0.500000 & 0.000000 & 0.500000 & $0.01140(3)$ \\
F1 & $0.71015(17)$ & $0.04893(6)$ & $0.45645(18)$ & $0.0254(3)$ \\
F2 & $0.31983(17)$ & $0.07728(6)$ & $0.39037(17)$ & $0.0232(2)$ \\
F3 & $0.58561(19)$ & $0.04104(7)$ & $0.74794(16)$ & $0.0274(3)$ \\
AG1 & $0.16018(2)$ & $0.12778(2)$ & $0.95234(2)$ & $0.01661(3)$ \\
N1 & $-0.1364(2)$ & $0.16467(9)$ & $0.8813(2)$ & $0.0179(3)$ \\
H1A & $-0.170(4)$ & $0.1789(14)$ & $0.968(4)$ & $0.029(7)^{*}$ \\
H1B & $-0.171(4)$ & $0.2005(14)$ & $0.805(4)$ & $0.028(7)^{*}$ \\
H1C & $-0.222(5)$ & $0.1286(19)$ & $0.805(5)$ & $0.070(11)^{*}$ \\
N2 & $0.4708(2)$ & $0.10144(9)$ & $1.0373(2)$ & $0.0175(3)$ \\
H2A & $0.509(4)$ & $0.0701(13)$ & $1.131(3)$ & $0.021(6)^{*}$ \\
H2B & $0.485(4)$ & $0.0811(15)$ & $0.935(4)$ & $0.041(8)^{*}$ \\
H2C & $0.557(5)$ & $0.1316(16)$ & $1.084(4)$ & $0.044(9)^{*}$ \\
N3 & $0.0911(3)$ & $0.00306(10)$ & $0.8291(3)$ & $0.0277(4)$ \\
H3A & $-0.031(5)$ & $-0.0050(15)$ & $0.751(5)$ & $0.046(9)^{*}$ \\
H3B & $0.149(5)$ & $-0.0181(16)$ & $0.920(5)$ & $0.043(9)^{*}$ \\
H3C & $0.133(5)$ & $-0.0059(15)$ & $0.737(4)$ & $0.043(9)^{*}$ \\
AG2 & $0.23394(2)$ & $0.22258(2)$ & $0.67497(2)$ & $0.01676(3)$ \\
N4 & $0.0292(3)$ & $0.15407(9)$ & $0.4859(2)$ & $0.0196(3)$ \\
H4A & $0.111(4)$ & $0.1257(14)$ & $0.459(4)$ & $0.033(7)^{*}$ \\
H4B & $-0.052(4)$ & $0.1757(14)$ & $0.396(4)$ & $0.034(7)^{*}$ \\
H4C & $-0.034(4)$ & $0.1288(15)$ & $0.520(4)$ & $0.033(8)^{*}$ \\
N5 & $0.4733(2)$ & $0.28292(9)$ & $0.8539(2)$ & $0.0167(3)$ \\
H5A & $0.543(4)$ & $0.2617(14)$ & $0.973(4)$ & $0.033(7)^{*}$ \\
H5B & $0.562(4)$ & $0.2883(12)$ & $0.793(3)$ & $0.020(6)^{*}$ \\
H5C & $0.439(5)$ & $0.3248(17)$ & $0.891(4)$ & $0.052(9)^{*}$ \\
F4 & $0.74284(16)$ & $0.21290(6)$ & $1.16824(14)$ & $0.0177(2)$ \\
& & & & \\
\hline & & & & \\
& & & & \\
& & & & \\
& & & & \\
\end{tabular}

Atomic displacement parameters $\left(\AA^{2}\right)$

\begin{tabular}{lllllll}
\hline & $U^{11}$ & $U^{22}$ & $U^{33}$ & $U^{12}$ & $U^{13}$ & $U^{23}$ \\
\hline SN1 & $0.01283(6)$ & $0.01077(7)$ & $0.01170(7)$ & $-0.00057(5)$ & $0.00600(5)$ & $-0.00041(5)$ \\
F1 & $0.0194(5)$ & $0.0291(7)$ & $0.0314(6)$ & $-0.0056(5)$ & $0.0139(5)$ & $0.0066(5)$ \\
F2 & $0.0207(5)$ & $0.0175(5)$ & $0.0319(6)$ & $0.0053(4)$ & $0.0110(5)$ & $0.0065(5)$ \\
F3 & $0.0329(6)$ & $0.0314(7)$ & $0.0179(5)$ & $-0.0038(5)$ & $0.0098(5)$ & $-0.0095(5)$
\end{tabular}




\begin{tabular}{lllllll} 
& & & & & \\
AG1 & $0.01565(5)$ & $0.01867(7)$ & $0.01611(6)$ & $0.00126(5)$ & $0.00689(4)$ & $0.00184(5)$ \\
N1 & $0.0179(7)$ & $0.0166(7)$ & $0.0183(7)$ & $0.0021(6)$ & $0.0061(6)$ & $0.0005(6)$ \\
N2 & $0.0175(6)$ & $0.0178(7)$ & $0.0160(7)$ & $0.0000(6)$ & $0.0053(5)$ & $-0.0007(6)$ \\
N3 & $0.0206(8)$ & $0.0236(9)$ & $0.0346(10)$ & $0.0001(7)$ & $0.0063(8)$ & $-0.0068(8)$ \\
AG2 & $0.01614(6)$ & $0.01801(7)$ & $0.01510(6)$ & $-0.00111(5)$ & $0.00505(4)$ & $0.00066(4)$ \\
N4 & $0.0184(7)$ & $0.0174(8)$ & $0.0194(7)$ & $-0.0010(6)$ & $0.0035(6)$ & $0.0012(6)$ \\
N5 & $0.0169(6)$ & $0.0173(7)$ & $0.0161(7)$ & $0.0003(5)$ & $0.0066(5)$ & $0.0011(5)$ \\
F4 & $0.0176(5)$ & $0.0200(5)$ & $0.0143(5)$ & $-0.0003(4)$ & $0.0051(4)$ & $-0.0015(4)$ \\
\hline
\end{tabular}

Geometric parameters $\left(\AA,{ }^{\circ}\right)$

\begin{tabular}{|c|c|c|c|}
\hline $\mathrm{Sn} 1-\mathrm{F} 1^{\mathrm{i}}$ & $1.9518(11)$ & $\mathrm{N} 2-\mathrm{H} 2 \mathrm{~A}$ & $0.91(3)$ \\
\hline $\mathrm{Sn} 1-\mathrm{F} 1$ & $1.9518(11)$ & $\mathrm{N} 2-\mathrm{H} 2 \mathrm{~B}$ & $0.94(3)$ \\
\hline $\mathrm{Sn} 1-\mathrm{F} 2$ & $1.9617(11)$ & $\mathrm{N} 2-\mathrm{H} 2 \mathrm{C}$ & $0.83(3)$ \\
\hline $\mathrm{Sn} 1-\mathrm{F} 2^{\mathrm{i}}$ & $1.9617(11)$ & $\mathrm{N} 3-\mathrm{H} 3 \mathrm{~A}$ & $0.88(3)$ \\
\hline $\mathrm{Sn} 1-\mathrm{F} 3^{\mathrm{i}}$ & $1.9655(11)$ & $\mathrm{N} 3-\mathrm{H} 3 \mathrm{~B}$ & $0.79(3)$ \\
\hline $\mathrm{Sn} 1-\mathrm{F} 3$ & $1.9656(11)$ & $\mathrm{N} 3-\mathrm{H} 3 \mathrm{C}$ & $0.91(3)$ \\
\hline $\operatorname{Ag} 1-\mathrm{N} 1$ & $2.1434(16)$ & $\mathrm{Ag} 2-\mathrm{N} 4$ & $2.1160(16)$ \\
\hline $\mathrm{Ag} 1-\mathrm{N} 2$ & $2.1662(16)$ & $\mathrm{Ag} 2-\mathrm{N} 5$ & $2.1183(16)$ \\
\hline Ag1-N3 & $2.5870(19)$ & $\mathrm{N} 4-\mathrm{H} 4 \mathrm{~A}$ & $0.90(3)$ \\
\hline $\mathrm{Ag} 1-\mathrm{Ag} 2$ & $3.0611(2)$ & $\mathrm{N} 4-\mathrm{H} 4 \mathrm{~B}$ & $0.84(3)$ \\
\hline $\mathrm{Ag} 1-\mathrm{Ag} 2^{\mathrm{ii}}$ & $3.3283(2)$ & $\mathrm{N} 4-\mathrm{H} 4 \mathrm{C}$ & $0.79(3)$ \\
\hline $\mathrm{N} 1-\mathrm{H} 1 \mathrm{~A}$ & $0.85(3)$ & $\mathrm{N} 5-\mathrm{H} 5 \mathrm{~A}$ & $0.96(3)$ \\
\hline N1-H1B & $0.89(3)$ & N5-H5B & $0.95(3)$ \\
\hline $\mathrm{N} 1-\mathrm{H} 1 \mathrm{C}$ & $0.98(4)$ & $\mathrm{N} 5-\mathrm{H} 5 \mathrm{C}$ & $0.93(3)$ \\
\hline $\mathrm{F} 1-\mathrm{S} n 1-\mathrm{F} 1$ & 180.0 & $\mathrm{Ag} 1-\mathrm{N} 2-\mathrm{H} 2 \mathrm{~A}$ & $110.8(15)$ \\
\hline $\mathrm{F} 1{ }^{\mathrm{i}}-\mathrm{Sn} 1-\mathrm{F} 2$ & $90.56(5)$ & $\mathrm{Ag} 1-\mathrm{N} 2-\mathrm{H} 2 \mathrm{~B}$ & $107.2(17)$ \\
\hline $\mathrm{F} 1-\mathrm{Sn} 1-\mathrm{F} 2$ & $89.44(5)$ & $\mathrm{H} 2 \mathrm{~A}-\mathrm{N} 2-\mathrm{H} 2 \mathrm{~B}$ & $108(2)$ \\
\hline $\mathrm{F} 1^{\mathrm{i}}-\mathrm{Sn} 1-\mathrm{F} 2^{\mathrm{i}}$ & $89.44(5)$ & $\mathrm{Ag} 1-\mathrm{N} 2-\mathrm{H} 2 \mathrm{C}$ & $119(2)$ \\
\hline $\mathrm{F} 1-\mathrm{Sn} 1-\mathrm{F} 2^{\mathrm{i}}$ & $90.56(5)$ & $\mathrm{H} 2 \mathrm{~A}-\mathrm{N} 2-\mathrm{H} 2 \mathrm{C}$ & $100(2)$ \\
\hline $\mathrm{F} 2-\mathrm{Sn} 1-\mathrm{F} 2^{\mathrm{i}}$ & 180.0 & $\mathrm{H} 2 \mathrm{~B}-\mathrm{N} 2-\mathrm{H} 2 \mathrm{C}$ & $111(3)$ \\
\hline $\mathrm{F} 1^{\mathrm{i}}-\mathrm{Sn} 1-\mathrm{F} 3^{\mathrm{i}}$ & $90.58(6)$ & $\mathrm{Ag} 1-\mathrm{N} 3-\mathrm{H} 3 \mathrm{~A}$ & $115(2)$ \\
\hline $\mathrm{F} 1-\mathrm{Sn} 1-\mathrm{F}^{\mathrm{i}}$ & $89.42(6)$ & $\mathrm{Ag} 1-\mathrm{N} 3-\mathrm{H} 3 \mathrm{~B}$ & $101(2)$ \\
\hline $\mathrm{F} 2-\mathrm{Sn} 1-\mathrm{F}^{\mathrm{i}}$ & $88.80(5)$ & $\mathrm{H} 3 \mathrm{~A}-\mathrm{N} 3-\mathrm{H} 3 \mathrm{~B}$ & $126(3)$ \\
\hline $\mathrm{F} 2^{\mathrm{i}}-\mathrm{Sn} 1-\mathrm{F} 3^{\mathrm{i}}$ & $91.19(5)$ & $\mathrm{Ag} 1-\mathrm{N} 3-\mathrm{H} 3 \mathrm{C}$ & 113.7 (19) \\
\hline $\mathrm{F} 1-\mathrm{S} n 1-\mathrm{F} 3$ & $89.42(6)$ & $\mathrm{H} 3 \mathrm{~A}-\mathrm{N} 3-\mathrm{H} 3 \mathrm{C}$ & $89(3)$ \\
\hline $\mathrm{F} 1-\mathrm{Sn} 1-\mathrm{F} 3$ & $90.58(6)$ & $\mathrm{H} 3 \mathrm{~B}-\mathrm{N} 3-\mathrm{H} 3 \mathrm{C}$ & $113(3)$ \\
\hline $\mathrm{F} 2-\mathrm{Sn} 1-\mathrm{F} 3$ & $91.19(5)$ & $\mathrm{N} 4-\mathrm{Ag} 2-\mathrm{N} 5$ & $170.93(7)$ \\
\hline $\mathrm{F} 2 \mathrm{i}-\mathrm{Sn} 1-\mathrm{F} 3$ & $88.81(5)$ & $\mathrm{N} 4-\mathrm{Ag} 2-\mathrm{Ag} 1$ & $81.11(5)$ \\
\hline $\mathrm{F} 3{ }^{\mathrm{i}}-\mathrm{Sn} 1-\mathrm{F} 3$ & 180.0 & $\mathrm{~N} 5-\mathrm{Ag} 2-\mathrm{Ag} 1$ & $101.15(5)$ \\
\hline $\mathrm{N} 1-\mathrm{Ag} 1-\mathrm{N} 2$ & $173.74(7)$ & $\mathrm{N} 4-\mathrm{Ag} 2-\mathrm{Ag} 1^{\mathrm{iii}}$ & $104.96(5)$ \\
\hline $\mathrm{N} 1-\mathrm{Ag} 1-\mathrm{N} 3$ & $100.82(6)$ & $\mathrm{N} 5-\mathrm{Ag} 2-\mathrm{Ag} 1^{\mathrm{iii}}$ & $77.58(4)$ \\
\hline $\mathrm{N} 2-\mathrm{Ag} 1-\mathrm{N} 3$ & $85.44(6)$ & $\mathrm{Ag} 1-\mathrm{Ag} 2-\mathrm{Ag} 1^{\mathrm{iii}}$ & $149.685(7)$ \\
\hline $\mathrm{N} 1-\mathrm{Ag} 1-\mathrm{Ag} 2$ & $93.35(5)$ & $\mathrm{Ag} 2-\mathrm{N} 4-\mathrm{H} 4 \mathrm{~A}$ & $101.3(17)$ \\
\hline $\mathrm{N} 2-\mathrm{Ag} 1-\mathrm{Ag} 2$ & $84.44(5)$ & $\mathrm{Ag} 2-\mathrm{N} 4-\mathrm{H} 4 \mathrm{~B}$ & $110.4(19)$ \\
\hline $\mathrm{N} 3-\mathrm{Ag} 1-\mathrm{Ag} 2$ & $111.20(6)$ & $\mathrm{H} 4 \mathrm{~A}-\mathrm{N} 4-\mathrm{H} 4 \mathrm{~B}$ & $115(3)$ \\
\hline $\mathrm{N} 1-\mathrm{Ag} 1-\mathrm{Ag} 2^{\mathrm{ii}}$ & $77.42(5)$ & $\mathrm{Ag} 2-\mathrm{N} 4-\mathrm{H} 4 \mathrm{C}$ & $120(2)$ \\
\hline
\end{tabular}




$\begin{array}{llll}\mathrm{N} 2-\mathrm{Ag} 1-\mathrm{Ag} 2^{\mathrm{ii}} & 96.40(5) & \mathrm{H} 4 \mathrm{~A}-\mathrm{N} 4-\mathrm{H} 4 \mathrm{C} & 104(3) \\ \mathrm{N} 3-\mathrm{Ag} 1-\mathrm{Ag} 2^{\mathrm{ii}} & 169.75(6) & \mathrm{H} 4 \mathrm{~B}-\mathrm{N} 4-\mathrm{H} 4 \mathrm{C} & 107(3) \\ \mathrm{Ag} 2-\mathrm{Ag} 1-\mathrm{Ag} 2^{\mathrm{ii}} & 79.041(4) & \mathrm{Ag} 2-\mathrm{N} 5-\mathrm{H} 5 \mathrm{~A} & 113.0(16) \\ \mathrm{Ag} 1-\mathrm{N} 1-\mathrm{H} 1 \mathrm{~A} & 118.6(17) & \mathrm{Ag} 2-\mathrm{N} 5-\mathrm{H} 5 \mathrm{~B} & 106.2(14) \\ \mathrm{Ag} 1-\mathrm{N} 1-\mathrm{H} 1 \mathrm{~B} & 115.3(18) & \mathrm{H} 5 \mathrm{~A}-\mathrm{N} 5-\mathrm{H} 5 \mathrm{~B} & 109(2) \\ \mathrm{H} 1 \mathrm{~A}-\mathrm{N} 1-\mathrm{H} 1 \mathrm{~B} & 101(2) & \mathrm{Ag} 2-\mathrm{N} 5-\mathrm{H} 5 \mathrm{C} & 115.7(19) \\ \mathrm{Ag} 1-\mathrm{N} 1-\mathrm{H} 1 \mathrm{C} & 105(2) & \mathrm{H} 5 \mathrm{~A}-\mathrm{N} 5-\mathrm{H} 5 \mathrm{C} & 100(2) \\ \mathrm{H} 1 \mathrm{~A}-\mathrm{N} 1-\mathrm{H} 1 \mathrm{C} & 114(3) & \mathrm{H} 5 \mathrm{~B}-\mathrm{N} 5-\mathrm{H} 5 \mathrm{C} & 112(2) \\ \mathrm{H} 1 \mathrm{~B}-\mathrm{N} 1-\mathrm{H} 1 \mathrm{C} & 102(3) & & \end{array}$

Symmetry codes: (i) $-x+1,-y,-z+1$; (ii) $x,-y+1 / 2, z+1 / 2$; (iii) $x,-y+1 / 2, z-1 / 2$.

Hydrogen-bond geometry $\left(\AA,{ }^{\circ}\right)$

\begin{tabular}{lllll}
\hline$D-\mathrm{H} \cdots A$ & $D-\mathrm{H}$ & $\mathrm{H} \cdots A$ & $D \cdots A$ & $D-\mathrm{H}^{\cdots} A$ \\
\hline $\mathrm{N} 1-\mathrm{H} 1 A \cdots \mathrm{F} 4^{\text {iv }}$ & $0.85(3)$ & $2.03(3)$ & $2.884(2)$ & $178(2)$ \\
$\mathrm{N} 1-\mathrm{H} 1 B \cdots \mathrm{F} 4^{\mathrm{v}}$ & $0.89(3)$ & $1.96(3)$ & $2.844(2)$ & $171(3)$ \\
$\mathrm{N} 1-\mathrm{H} 1 C \cdots \mathrm{F} 3^{\text {iv }}$ & $0.98(4)$ & $2.14(4)$ & $3.057(2)$ & $156(3)$ \\
$\mathrm{N} 2-\mathrm{H} 2 A \cdots \mathrm{F} 1^{\text {vi }}$ & $0.91(3)$ & $2.43(3)$ & $3.227(2)$ & $146(2)$ \\
$\mathrm{N} 2-\mathrm{H} 2 A \cdots \mathrm{F} 3^{\text {vii }}$ & $0.91(3)$ & $2.56(3)$ & $3.354(2)$ & $145.4(19)$ \\
$\mathrm{N} 2-\mathrm{H} 2 B \cdots \mathrm{F} 3$ & $0.94(3)$ & $2.04(3)$ & $2.961(2)$ & $167(3)$ \\
$\mathrm{N} 2-\mathrm{H} 2 C \cdots \mathrm{F} 4$ & $0.83(3)$ & $2.02(3)$ & $2.849(2)$ & $172(3)$ \\
$\mathrm{N} 3-\mathrm{H} 3 A \cdots \mathrm{F} 1^{\text {iv }}$ & $0.88(3)$ & $2.57(3)$ & $3.274(2)$ & $138(3)$ \\
$\mathrm{N} 3-\mathrm{H} 3 A \cdots \mathrm{F} 2^{\text {viii }}$ & $0.88(3)$ & $2.42(3)$ & $3.223(2)$ & $153(3)$ \\
$\mathrm{N} 3-\mathrm{H} 3 B \cdots \mathrm{F} 3^{\text {vii }}$ & $0.79(3)$ & $2.61(3)$ & $3.345(3)$ & $157(3)$ \\
$\mathrm{N} 3-\mathrm{H} 3 C \cdots \mathrm{F} 1^{\text {i }}$ & $0.91(3)$ & $2.39(3)$ & $3.279(3)$ & $167(3)$ \\
$\mathrm{N} 4-\mathrm{H} 4 A \cdots \mathrm{F} 2$ & $0.90(3)$ & $2.04(3)$ & $2.930(2)$ & $170(2)$ \\
$\mathrm{N} 4-\mathrm{H} 4 B \cdots \mathrm{F} 4^{\text {ix }}$ & $0.84(3)$ & $1.97(3)$ & $2.7955(19)$ & $171(3)$ \\
$\mathrm{N} 4-\mathrm{H} 4 C \cdots \mathrm{F} 1^{\text {iv }}$ & $0.79(3)$ & $2.33(3)$ & $3.045(2)$ & $151(3)$ \\
$\mathrm{N} 5-\mathrm{H} 5 A \cdots \mathrm{F} 4$ & $0.96(3)$ & $1.90(3)$ & $2.8305(19)$ & $160(3)$ \\
$\mathrm{N} 5-\mathrm{H} 5 B \cdots \mathrm{F} 4^{\text {iii }}$ & $0.95(3)$ & $1.93(3)$ & $2.882(2)$ & $173(2)$ \\
$\mathrm{N} 5-\mathrm{H} 5 C \cdots \mathrm{F} 2^{\mathrm{ii}}$ & $0.93(3)$ & $2.09(3)$ & $2.999(2)$ & $163(3)$ \\
& & & &
\end{tabular}

Symmetry codes: (i) $-x+1,-y,-z+1$; (ii) $x,-y+1 / 2, z+1 / 2$; (iii) $x,-y+1 / 2, z-1 / 2$; (iv) $x-1, y, z$; (v) $x-1,-y+1 / 2, z-1 / 2$; (vi) $x, y, z+1$; (vii) $-x+1,-y,-z+2$; (viii) $-x,-y,-z+1 ;$ (ix) $x-1, y, z-1$. 